\title{
An Eigen-Based TEQ Design for VDSL Systems
}

\author{
Yuan-Pei Lin, Senior Member, IEEE, Li-Han Liang, Pei-Ju Chung, and See-May Phoong, Member, IEEE
}

\begin{abstract}
In this paper, we propose an eigen-based semiblind time-domain equalizer (TEQ) design method for VDSL systems. We exploit the fact that the VDSL system is frequency-division duplexing and maximize signal-to-interference ratio in the frequency domain. Unlike earlier eigen-based TEQ designs, the proposed method does not require the channel impulse response nor channel noise statistics. Using properties of the training symbols in VDSL initialization, the TEQ can be obtained by computing an eigenvector of a positive definite matrix that depends only on the averaged received VDSL symbols. The proposed method is referred to as semiblind, as properties of the training symbols are used but the channel impulse response is not known. Examples will be given to demonstrate that the proposed TEQ design method can effectively shorten the channel impulse response and achieve very good bit rates with only a small number of training symbols.
\end{abstract}

Index Terms-Discrete multitone (DMT), eigen approach, frequency-based singal-to-interference ratio (SIR), multicarrier, semiblind, time-domain equalizer (TEQ), VDSL.

\section{INTRODUCTION}

$\mathbf{T}$ HE discrete Fourier transform (DFT)-based discrete multitone (DMT) transceiver has found important applications in digital subscriber loop (DSL) [1], [2]. The transmitter and receiver perform, respectively, $M$-point inverse DFT (IDFT) and DFT computations, where $M$ is the number of tones or number of subchannels. At the transmitter side, each block is prefixed with a cyclic prefix of length $L$; the last $L$ samples of each block are copied to the beginning of the block. If $L$ is no smaller than the order of the channel, then interblock interference (IBI) can be removed easily by discarding the prefix at the receiver. As a result, a finite impulse response (FIR) channel is converted into $M$ frequency nonselective parallel subchannels. The subchannel gains are the $M$-point DFT of the channel impulse response. When the channel is longer or much longer than $L$, which is usually the case in DSL applications, a time-domain

Manuscript received January 5, 2005; accepted January 13, 2006. This work was supported in part by NSC 94-2213-E-009-038, NSC94-2752-E-002-006PAE, and NSC94-2213-E-002-0753, Taiwan, R.O.C. The associate editor coordinating the review of this manuscript and approving it for publication was Dr. Luc Vandendorpe.

Y.-P. Lin is with the Department of Electrical and Control Engineering, National Chiao Tung University, Hsinchu, Taiwan, R.O.C. (e-mail: ypl@cc.nctu. edu.tw).

L.-H. Liang was with the Department of Electrical and Control Engineering, National Chiao Tung University, Hsinchu, Taiwan, R.O.C. He is now with REALTEK Seminconductor Corporation, Taiwan, R.O.C.

P.-J. Chung was with the Department of Electrical and Control Engineering, National Chiao Tung University, Hsinchu, Taiwan, R.O.C. She is now with the Department of Communications Engineering, National Chiao Tung University, Hsinchu, Taiwan, R.O.C.

S.-M. Phoong is with the Department of Electrical Engineering, Graduate Institute of Communications Engineering, National Taiwan University, Taipei, Taiwan, R.O.C. (e-mail: smp@cc.ee.ntu.edu.tw).

Digital Object Identifier 10.1109/TSP.2006.882116 equalizer (TEQ) is usually inserted at the receiver to shorten the channel impulse response so that the equivalent channel has most of the energy concentrated in a window of $L+1$ samples [3]. The samples outside the window, however, will lead to IBI, which reduces signal-to-(interference and noise) ratio (SINR) and thus affects transmission bit rate. The time-domain equalizers play an important role in the application of DMT to DSL [1], [2]. The bit rates that can be transmitted are greatly affected by the design of TEQ.

Many TEQ designs have been proposed in the literature. In [4]-[8], a TEQ that maximizes the transmission bit rate is considered. For bit rate maximization, nonlinear optimization is often needed in the search of an optimal TEQ and a globally optimal solution is not guaranteed. In many of the existing methods, optimal TEQs, of different design criteria, are computed once the channel impulse response and channel noise statistics are given [3]-[10]. In [3]-[12], the TEQ design problem is formulated as an eigenfilter problem and optimal TEQ in a certain sense can be designed by computing an eigenvector corresponding to an appropriately defined channel dependent matrix. In [3], Melsa et al.. designed the optimal TEQ with minimum IBI. The ratio of the energy of the equivalent channel in a window of $L+1$ samples over the energy outside the window is maximized. This approach is called maximum shortening signal-to-noise ratio (MSSNR). By incorporating noise statistics in MSSNR in [3], a higher bit rate can be achieved [9]. A low-complexity channel shortening equalizer that requires only one Cholesky decomposition for all window positions is given in [10]. A TEQ objective function independent of the position of the window is proposed in [11].

In [12], zero padding is applied in the frequency domain to impose extra null symbols. Using a quadratic objective function based on the null symbols, blind adaptive equalization that does not require the channel impulse response can be achieved. The equalizer designed in [12] is different from the usual TEQ for DMT systems in the sense that the goal is to have ideal equalization so that the equivalent channel has only one tap. In [13], the TEQ is designed by linearly combining the eigenvectors of an appropriately defined matrix that depends on the channel and channel noise. The parameter that determines how the eigenvectors are linearly combined may be found experimentally. In these methods, the TEQ depends directly on the channel impulse response, an estimate of which is needed using training symbols before TEQ optimization can take place. In [14], Martin et al.. proposed multicarrier equalization by restoration of redundancy (MERRY). MERRY is a blind adaptive algorithm that exploits cyclic prefix to design the TEQ. It is shown in [14] to be a globally convergent algorithm. A comprehensive overview on TEQs and a unified design approach is available in [15].

In this paper, we propose a TEQ design method for the VDSL system that maximizes signal-to-interference ratio (SIR) in the 
frequency domain using an eigenfilter approach. In the VDSL system, frequency-division duplex is used to separate upstream and downstream signals. In symmetric transmission mode, around half the tones are set aside for downstream transmission and the other half for upstream. For asymmetric transmission mode, more tones are used for downstream and fewer for upstream. For the downstream application, the upstream tones are referred to as the null tones in this paper. Similarly, the downstream tones are called the null tones in the upstream transmission.

In VDSL training symbols, around half of the downstream/upstream tones are pilots and the other half carry messages [2]. We will exploit these properties of the training symbols to maximize SIR in the frequency domain. Our proposed method is different from existing eigenmethods in two aspects. First, the channel impulse response is not needed and the TEQ can be computed directly using an average of the received VDSL symbols without channel estimation. Otherwise an estimate of the channel impulse response should be first computed for TEQ optimization and the performance depends on the accuracy of channel estimation. An estimate of the channel impulse response is more complex for VDSL systems. as not all the tones are used and the frequency response of the channel is available only on the tones used. Furthermore, not having to estimate the channel will also save training time. Secondly, the objective function is formulated in the frequency domain; the optimization implicitly takes into account the equivalent channel's frequency response. The frequency response of the equivalent channel critically affects subchannel SNR and thus bit rate. We will demonstrate through examples that the proposed TEQ design method can achieve very good bit rates with only a small number of training symbols. Some preliminary results have been reported in [16].

This paper is organized as follows. In Section II, we give a brief review of training symbols in VDSL systems and mention the properties that will be used later for TEQ design. In Section III, we formulate an objective function of SIR in the frequency domain by exploiting the fact that the underlying system is frequency-division multiplexing and by exploiting properties of training symbols. The optimal TEQ is derived in Section IV. Simulation examples are given in Section V. A conclusion is given in Section VI.

\section{Notation:}

1) Boldfaced lower case letters represent vectors and boldfaced upper case letters are reserved for matrices. The notation $\mathbf{A}^{\dagger}$ denotes transpose conjugate of $\mathbf{A}$.

2) The notation $\mathbf{I}_{M}$ is used to represent the $M \times M$ identity matrix.

3) The notation $\mathbf{W}$ is used to represent the $M \times M$ unitary DFT matrix given by

$$
[\mathbf{W}]_{k n}=\frac{1}{\sqrt{M}} e^{-j \frac{2 \pi}{M} k n} \quad \text { for } 0 \leq k, n \leq M-1 .
$$

\section{VDSL SYSTEM TRAINING SYMBOLS}

In the VDSL system, frequency-division duplexing is used to separate upstream and downstream signals [2]. In symmetric transmission mode, around half the tones are set aside for downstream transmission and the other half for upstream transmission. For asymmetric transmission mode, more tones are used for downstream and fewer for upstream. For downstream application, the tones used for transmission are called the data tones and the upstream tones are called the null tones. On the null tones, zeros are sent. Similarly, in upstream applications, the tones used for transmission are the data tones and the downstream tones are the null tones. In one of the training stages, only one direction is transmitting while the other direction is silent, i.e., not transmitting anything. In this stage, we can assume the signals on the null tones are zeros. Note that the null tones may contain the signal transmitted in the opposite direction in "show time," the stage of actual data transmission. In a VDSL training symbol, about half of the data tones are set aside for pilots (even tones and tones that are multiples of ten plus nine) and the others are used for transmitting initialization messages. For all data tones, symbols are drawn from a quadrature phase-shift keying (QPSK) constellation. The QPSK symbols on the pilot tones are determined in a pseudorandom manner but remain the same for all training symbols. The QPSK symbols on message tones vary with training symbols. Therefore, the $n$th input vector of the IDFT matrix on the transmitter side can be written in the form

$$
\mathbf{s}(n)=\mathbf{P}\left(\begin{array}{c}
\mathbf{s}_{p} \\
\mathbf{s}_{m}(n) \\
\mathbf{0}
\end{array}\right)
$$

where $\mathbf{s}_{p}$ is a constant vector consisting of QPSK symbols on pilot tones, $\mathbf{s}_{m}(n)$ is a time-dependent vector consisting of QPSK symbols on message tones, and $\mathbf{P}$ is a permutation matrix. The matrix $\mathbf{P}$ is included for notational convenience as the actual pilot tone indices and message tone indexes are interleaved. The symbols on the null tones are zeros.

When the channel order is no larger than the length of the cyclic prefix $L$, we know there is no IBI after removing guard samples (prefix removal). In the absence of channel noise, the outputs of the DFT matrix at the receiving end are the scaled versions of the transmitter inputs. The scalars are the $M$-point DFT of the channel impulse response. The null tones will be nothing but channel noise if transmission in the opposite direction is idle. However, if the channel order is larger than the length of cyclic prefix, there will be IBI even after removing guard samples. The outputs of the null tones now have channel noise plus interference from the data tones of the previous block due to IBI (assuming the channel order is smaller than $N=M+L$ ). Our proposed method will exploit the fact that the symbols sent on pilot tones and the null tones are fixed in each VDSL training symbol to formulate a quadratic objective function of SIR in the frequency domain for TEQ optimization.

\section{OBjective Function: Signal to InTERfERENCE RATIO}

In this section, we derive the objective function to be used in the proposed TEQ design method. Fig. 1 shows the block diagram of a DMT transceiver. Using matrix representation for cyclic prefix insertion and prefix removal, Fig. 1 can be redrawn 


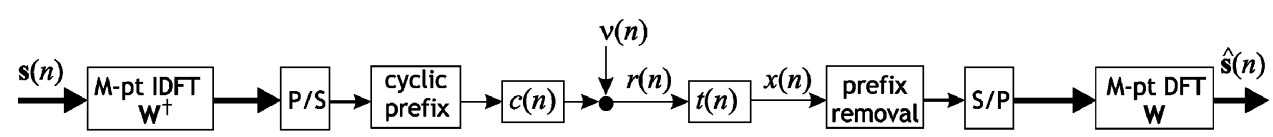

Fig. 1. The DMT transceiver.

(a)

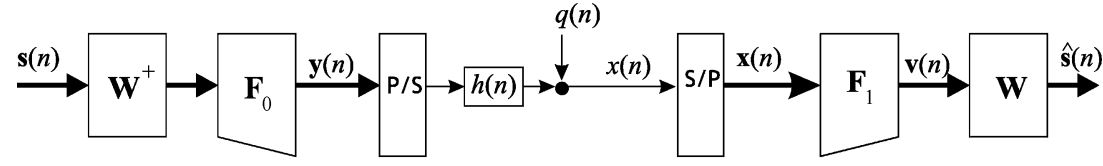

$q(n)$

(b)

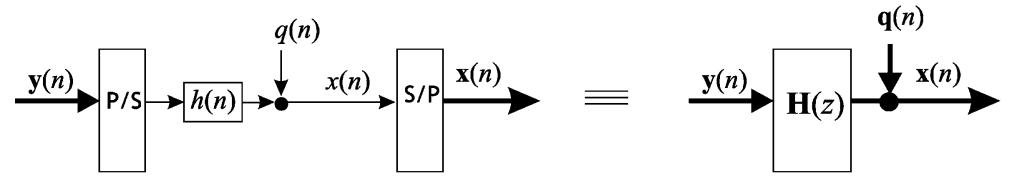

(c)

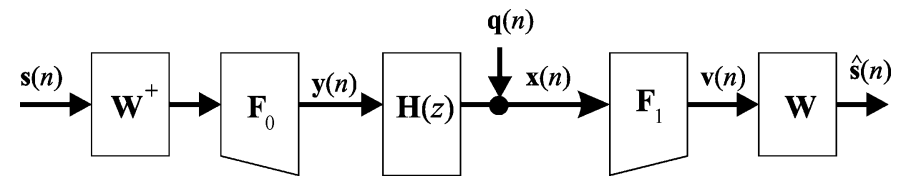

Fig. 2. Equivalent block diagrams of the DMT transceiver.

as Fig. 2(a), where the matrices $\mathbf{F}_{0}$ and $\mathbf{F}_{1}$ are, respectively, of dimensions $N \times M$ and $M \times N$

$$
\mathbf{F}_{0}=\left(\begin{array}{cc}
\mathbf{0} & \mathbf{I}_{L} \\
\mathbf{I}_{M}
\end{array}\right), \quad \mathbf{F}_{1}=\left(\begin{array}{ll}
\mathbf{0} & \mathbf{I}_{M}
\end{array}\right) .
$$

The matrix $\mathbf{F}_{0}$ carries out the operation of prefix insertion whereas $\mathbf{F}_{1}$ discards the prefix. The "P/S" operation converts $M$ parallel inputs to a serial output and the "S/P" operation converts a serial input to $M$ parallel outputs. In Fig. 2(a), we have lumped the channel $c(n)$ and the equalizer $t(n)$ together as $h(n)=c(n) * t(n)$, and $q(n)$ is the noise after TEQ, $q(n)=\nu(n) * t(n)$. At the receiver side in Fig. 2(a), the input vector $\mathbf{x}(n)$ of the matrix $\mathbf{F}_{1}$ is given by

$$
\mathbf{x}(n)=\left(\begin{array}{c}
x(n N) \\
x(n N+1) \\
\vdots \\
x(n N+N-1)
\end{array}\right) .
$$

(In the above equation, we have assumed the VDSL symbols have been perfectly synchronized. Otherwise, the received vector has an extra delay parameter $d$; in this case, $\mathbf{x}(n)$ becomes $\mathbf{x}(n)=(x(n N+d) x(n N+1+d) \quad \ldots x(n N+$ $N-1+d))^{T}$.) The output vector $\mathbf{v}(n)$ of $\mathbf{F}_{1}$ is obtained from $\mathbf{x}(n)$ by removing the first $L$ samples

$$
\mathbf{v}(n)=\left(\begin{array}{c}
x(n N+L) \\
x(n N+L+1) \\
\vdots \\
x(n N+N-1)
\end{array}\right)
$$

If we disregard the channel noise, the $N \times N$ system from $\mathbf{y}(n)$ to $\mathbf{x}(n)$ is a linear time-invariant system and the transfer matrix $\mathbf{H}(z)$ is pseudocirculant [17]. Assuming the length of $h(n)$ is no longer than $N=M+L$, then the transfer matrix $\mathbf{H}(z)$ is [17]

$$
\left(\begin{array}{ccccc}
h(0) & z^{-1} h(N-1) & z^{-1} h(N-2) & \cdots & z^{-1} h(1) \\
h(1) & h(0) & z^{-1} h(N-1) & \cdots & z^{-1} h(2) \\
h(2) & h(1) & h(0) & \cdots & z^{-1} h(3) \\
\vdots & \vdots & \vdots & \ddots & \vdots \\
h(N-1) & h(N-2) & h(N-3) & \cdots & h(0)
\end{array}\right) .
$$

As $\mathbf{H}(z)$ is of order one, we can express it as

$$
\mathbf{H}(z)=\mathbf{H}_{0}+z^{-1} \mathbf{H}_{1}
$$

where

$$
\begin{aligned}
\mathbf{H}_{0} & =\left(\begin{array}{cccc}
h(0) & 0 & \cdots & 0 \\
h(1) & h(0) & \cdots & 0 \\
\vdots & \vdots & \ddots & \vdots \\
h(N-1) & h(N-2) & \cdots & h(0)
\end{array}\right) \\
\mathbf{H}_{1} & =\left(\begin{array}{cccc}
0 & h(N-1) & \cdots & h(1) \\
0 & 0 & \cdots & h(2) \\
\vdots & \vdots & \ddots & \vdots \\
0 & 0 & \cdots & 0
\end{array}\right) .
\end{aligned}
$$

The part of Fig. 2(a) from $\mathbf{y}(n)$ to $\mathbf{x}(n)$ can be redrawn as in Fig. 2(b), where $\mathbf{q}(n)$ is the $N \times 1$ channel noise vector blocked from $q(n)$. Using this matrix representation, we can further redraw the system in Fig. 2(a) as in Fig. 2(c). The vector $\mathbf{y}(n)$ shown in Fig. 2(c) is given by

$$
\mathbf{y}(n)=\mathbf{F}_{0} \mathbf{W}^{\dagger} \mathbf{s}(n)=\mathbf{F}_{0} \mathbf{W}^{\dagger} \mathbf{P}\left(\begin{array}{c}
\mathbf{s}_{p} \\
\mathbf{s}_{m}(n) \\
\mathbf{0}
\end{array}\right)
$$

where a permutation matrix $\mathbf{P}$ is included so that the input vector can be conveniently expressed as the pilot vector followed by the message vector and the null vector. 
The received vector $\mathbf{x}(n)$ can be written as

$$
\mathbf{x}(n)=\mathbf{H}_{0} \mathbf{y}(n)+\mathbf{H}_{1} \mathbf{y}(n-1)+\mathbf{q}(n) .
$$

The output vector of the receiver is $\widehat{\mathbf{s}}(n)=\mathbf{W F}_{1} \mathbf{x}(n)$. For convenience, we permute the outputs into the order of pilot tones followed by message tones and null tones. Let the receiver output vector after permutation be $\mathbf{u}(n)=\mathbf{P}^{T} \widehat{\mathbf{s}}(n)$. Then

$$
\mathbf{u}(n)=\mathbf{P}^{T} \mathbf{W} \mathbf{F}_{1} \mathbf{x}(n)=\mathbf{P}^{T} \mathbf{W} \mathbf{v}(n) .
$$

Suppose we collect $B$ vectors of $\mathbf{u}(n)$ and let

$$
\overline{\mathbf{u}}=\frac{1}{B} \sum_{n=1}^{B} \mathbf{u}(n)
$$

We also let

$$
\overline{\mathbf{v}}=\frac{1}{B} \sum_{n=1}^{B} \mathbf{v}(n), \quad \overline{\mathbf{q}}=\frac{1}{B} \sum_{n=1}^{B} \mathbf{q}(n) .
$$

The averaged vector $\overline{\mathbf{u}}$ is related to $\overline{\mathbf{v}}$ by $\overline{\mathbf{u}}=\mathbf{P}^{T} \mathbf{W} \overline{\mathbf{v}}$. Using (4) and the expression of $\mathbf{y}(n)$ in (3), we have

$$
\begin{aligned}
\overline{\mathbf{v}}= & \mathbf{F}_{1} \mathbf{H}_{0} \mathbf{F}_{0} \mathbf{W}^{\dagger} \mathbf{P}\left(\begin{array}{c}
\left.\frac{1}{B} \sum_{n=1}^{B} \mathbf{s}_{p} \mathbf{s}_{m}(n)\right) \\
\mathbf{0}
\end{array}\right) \\
& +\mathbf{F}_{1} \mathbf{H}_{1} \mathbf{F}_{0} \mathbf{W}^{\dagger} \mathbf{P}\left(\begin{array}{c}
\frac{1}{B} \sum_{n=1}^{B} \underset{\mathbf{0}}{\mathbf{s}_{p}} \mathbf{s}_{m}(n-1)
\end{array}\right)+\mathbf{F}_{1} \overline{\mathbf{q}} .
\end{aligned}
$$

Assume, reasonably, that the noise is zero mean and that the message is zero mean; then

$$
\overline{\mathbf{q}} \approx \mathbf{0}, \quad \text { and } \quad \frac{1}{B} \sum_{n=1}^{B} \mathbf{s}_{m}(n) \approx \mathbf{0} .
$$

(The zero-mean assumption on the noise is strengthened when the analog front-end bandpass filter that rejects the dc component is included.) We have

$$
\overline{\mathbf{v}} \approx \mathbf{F}_{1}\left(\mathbf{H}_{0}+\mathbf{H}_{1}\right) \mathbf{F}_{0} \mathbf{W}^{\dagger} \mathbf{P}\left(\begin{array}{c}
\mathbf{s}_{p} \\
\mathbf{0}
\end{array}\right) \text {. }
$$

Notice that $\mathbf{H}_{0}+\mathbf{H}_{1}$ is an $N$ by $N$ circulant matrix and is equal to

$$
\mathbf{H}_{0}+\mathbf{H}_{1}=\left.\mathbf{H}(z)\right|_{z=1}
$$

where $\mathbf{H}(z)$ is as defined in (2). Using this expression and the approximation of $\overline{\mathbf{v}}$ in (7), the averaged output vector $\overline{\mathbf{u}}$ can be approximated by

$$
\overline{\mathbf{u}} \approx \mathbf{P}^{T} \mathbf{W} \mathbf{F}_{1} \mathbf{H}(1) \mathbf{F}_{0} \mathbf{W}^{\dagger} \mathbf{P}\left(\begin{array}{c}
\mathbf{s}_{p} \\
\mathbf{0}
\end{array}\right) .
$$

Let us express $\mathbf{H}(1)$ as $\mathbf{C}_{0}+\mathbf{C}_{1}$, where $\mathbf{C}_{0}$ depends only on $h(0), h(1), \ldots, h(L)$ and $\mathbf{C}_{1}$ depends on the rest of the coefficients. As $\mathbf{H}(1)$ is circulant, the $N$ by $N$ matrix $\mathbf{C}_{0}$ is circulant with the first column given by

$$
\left(\begin{array}{lllllll}
h(0) & h(1) & \cdots & h(L) & 0 & \cdots & 0
\end{array}\right)^{T} .
$$

The matrix $\mathbf{C}_{1}$ is also an $N$ by $N$ circulant matrix with the first column given by

$$
\left(\begin{array}{lllllll}
0 & \cdots & 0 & h(L+1) & h(L+2) & \cdots & h(N-1)
\end{array}\right)^{T} .
$$

Then $\overline{\mathbf{u}}$ can be expressed as

$\overline{\mathbf{u}} \approx \mathbf{P}^{T} \mathbf{W F} \mathbf{F}_{1} \mathbf{C}_{0} \mathbf{F}_{0} \mathbf{W}^{\dagger} \mathbf{P}\left(\begin{array}{c}\mathbf{s}_{p} \\ \mathbf{0}\end{array}\right)+\underbrace{\mathbf{P}^{T} \mathbf{W F} \mathbf{F}_{1} \mathbf{C}_{1} \mathbf{F}_{0} \mathbf{W}^{\dagger} \mathbf{P}}_{\mathbf{C}_{1}^{\prime}}\left(\begin{array}{c}\mathbf{s}_{p} \\ \mathbf{0}\end{array}\right)$.

The first term represents the desired signal and the second term represents intersymbol interference (ISI). Notice that $\mathbf{F}_{1} \mathbf{C}_{0} \mathbf{F}_{0}$ is an $M \times M$ circulant matrix with the first column given by

$$
(h(0) \quad h(1) \quad \cdots \quad h(L) \quad 0 \quad \cdots \quad)^{T} .
$$

As an $M$ by $M$ DFT matrix diagonalizes all $M \times M$ circulant matrices, the product $\mathbf{W} \mathbf{F}_{1} \mathbf{C}_{0} \mathbf{F}_{0} \mathbf{W}^{\dagger}$ is a diagonal matrix $\Lambda$. The diagonal terms of $\Lambda$ are the $M$-point DFT of $h(0), h(1), \cdots h(L)$. As premultiplying by $\mathbf{P}^{T}$ and postmultiplying by $\mathbf{P}$ merely rearranges the diagonal terms of a diagonal matrix, the matrix $\mathbf{P}^{T}\left(\mathbf{W} \mathbf{F}_{1} \mathbf{C}_{0} \mathbf{F}_{0} \mathbf{W}^{\dagger}\right) \mathbf{P}=\mathbf{P}^{T} \Lambda \mathbf{P}$ continues to be a diagonal matrix $\Lambda^{\prime}=\mathbf{P}^{T} \boldsymbol{\Lambda} \mathbf{P}$. When the equivalent channel $h(n)$ has only $L+1$ coefficients, there is no IBI, $\mathbf{C}_{1}=\mathbf{0}$, and $\overline{\mathbf{u}}$ becomes

$$
\overline{\mathbf{u}} \approx \Lambda^{\prime}\left(\begin{array}{c}
\mathbf{s}_{p} \\
\mathbf{0}
\end{array}\right) .
$$

The output of the null tones and message tones is approximately zero and free from IBI. If the equivalent channel has more than $L+1$ coefficients, there will be IBI. Then from (8), we have

$$
\overline{\mathbf{u}} \approx \Lambda^{\prime}\left(\begin{array}{c}
\mathbf{s}_{p} \\
\mathbf{0}
\end{array}\right)+\mathbf{C}_{1}^{\prime}\left(\begin{array}{c}
\mathbf{s}_{p} \\
\mathbf{0}
\end{array}\right)
$$

where $\mathbf{C}_{1}^{\prime}=\mathbf{P}^{T} \mathbf{W} \mathbf{F}_{1} \mathbf{C}_{1} \mathbf{F}_{0} \mathbf{W}^{\dagger} \mathbf{P}$ as indicated in (8). Let us partition $\overline{\mathbf{u}}$ as

$$
\overline{\mathbf{u}}=\left(\begin{array}{c}
\overline{\mathbf{u}}_{p} \\
\overline{\mathbf{u}}_{m} \\
\overline{\mathbf{u}}_{n}
\end{array}\right)
$$

where $\overline{\mathbf{u}}_{p}, \overline{\mathbf{u}}_{m}$, and $\overline{\mathbf{u}}_{n}$ correspond to the outputs of pilot tones, message tones, and null tones, respectively. If we observe (9), we see that the average outputs $\overline{\mathbf{u}}_{n}$ on the null tones contain mostly interference from the pilot tones, as the first term on the right-hand side of (9) does not contribute to null tones. We propose the following objective function of frequency-domain SIR, which is the pilot tone energy over interference in the null tones

$$
\phi=\frac{\overline{\mathbf{u}}_{p}^{\dagger} \overline{\mathbf{u}}_{p}}{\overline{\mathbf{u}}_{n}^{\dagger} \overline{\mathbf{u}}_{n}} .
$$

Although $\overline{\mathbf{u}}_{p}^{\dagger} \overline{\mathbf{u}}_{p}$ contains pilot energy as well as terms due to interference, we use it to reflect pilot energy as interference is usually much smaller by comparison. Notice that the SIR in (10) is different from the conventional SIR in the time domain. The SIR in (10) is computed in the frequency domain. It is the signal 
power in the transmission band over the interference in the null tones. We will optimize the TEQ coefficients to maximize $\phi$.

Remarks:

- In the above formulation of objective function, we first average the received vectors and then compute the power on pilot tones and null tones, instead of the other way around. The reason for averaging first is to reduce the channel noise so that the average vector can give a good measure of interference. If we were to use the average energy in the null tones, i.e., $(1 / B) \sum_{i=1}^{B} \mathbf{u}_{n}^{\dagger}(i) \mathbf{u}_{n}(i)$, the term will contain both interference and noise in the null tones. But noise in the null tones is not relevant and need not be included.

- A possible TEQ optimization is to minimize $\overline{\mathbf{u}}_{n}^{\dagger} \overline{\mathbf{u}}_{n}$, which represents the interference present in the null tones. However, the final transmission bit rate is intrinsically related to the signal power in the tones used. The objective function in (10) incorporates signal power in the transmission bands and consequently leads to a better bit rate than that with the minimization of interference in null tones alone. Another possibility is to include the term $\mathbf{u}_{m}^{\dagger} \mathbf{u}_{m}$ in the denominator, which represents the interference in the message tones. The resulting objective function becomes

$$
\phi=\frac{\overline{\mathbf{u}}_{p}^{\dagger} \overline{\mathbf{u}}_{p}}{\overline{\mathbf{u}}_{m}^{\dagger} \overline{\mathbf{u}}_{m}+\overline{\mathbf{u}}_{n}^{\dagger} \overline{\mathbf{u}}_{n}} .
$$

\section{OPTIMAL TEQ DESIGN}

In what follows, we will see that the numerator and denominator of the objective function in (10) can be formulated as quadratic terms of the TEQ coefficients and the problem can be solved elegantly by computing an eigenvector of an appropriately defined positive definite matrix. Suppose the TEQ has order $Q, T(z)=\sum_{\ell=0}^{Q} t(\ell) z^{-\ell}$. The output of the TEQ can be written as

$$
x(n)=\sum_{\ell=0}^{Q} t(\ell) r(n-\ell)
$$

where $r(n)$ is the channel output as indicated in Fig. 1. The $M \times 1$ output vector $\mathbf{v}(n)$ of the matrix $\mathbf{F}_{1}$ given in (1) can be written in terms of TEQ coefficients as

$$
\mathbf{v}(n)=\mathbf{R}_{n} \mathbf{t}
$$

where $\mathbf{R}_{n}$ is an $M \times(Q+1)$ Toeplitz matrix given by the equation shown at the bottom of the page) and $\mathbf{t}$ is a column vector, $\mathbf{t}=(t(0) t(1) \cdots t(Q))^{T}$. The averaged vector in (5) is thus

$$
\overline{\mathbf{v}}=\overline{\mathbf{R}} \mathbf{t}, \quad \text { where } \quad \overline{\mathbf{R}}=\frac{1}{B} \sum_{n=1}^{B} \mathbf{R}_{n} .
$$

Therefore the averaged output vector $\overline{\mathbf{u}}$ is given by $\overline{\mathbf{u}}=\mathbf{P}^{T} \mathbf{W} \overline{\mathbf{R}} \mathbf{t}$. We partition the matrix $\mathbf{P}^{T} \mathbf{W} \overline{\mathbf{R}}$ as

$$
\mathbf{P}^{T} \mathbf{W} \overline{\mathbf{R}}=\left(\begin{array}{c}
\mathbf{A}_{p} \\
\mathbf{A}_{m} \\
\mathbf{A}_{n}
\end{array}\right)
$$

such that

$$
\left(\begin{array}{c}
\overline{\mathbf{u}}_{p} \\
\overline{\mathbf{u}}_{m} \\
\overline{\mathbf{u}}_{n}
\end{array}\right)=\left(\begin{array}{c}
\mathbf{A}_{p} \\
\mathbf{A}_{m} \\
\mathbf{A}_{n}
\end{array}\right) \mathbf{t} .
$$

The objective function given in (10) becomes

$$
\phi=\frac{\mathbf{t}^{\dagger} \mathbf{A}_{p}^{\dagger} \mathbf{A}_{p} \mathbf{t}}{\mathbf{t}^{\dagger} \mathbf{A}_{n}^{\dagger} \mathbf{A}_{n} \mathbf{t}} \text {. }
$$

The problem of maximizing $\phi$ in (10) is equivalent to

$$
\text { maximizing } \mathbf{t}^{\dagger} \mathbf{A}_{p}^{\dagger} \mathbf{A}_{p} \mathbf{t} \text {, subject to } \mathbf{t}^{\dagger} \mathbf{A}_{n}^{\dagger} \mathbf{A}_{n} \mathbf{t}=1 \text {. }
$$

The solution of the equivalent problem can be obtained by solving the generalized eigenvector problem [15], [18]. The optimal $\mathbf{t}$ is the eigenvector corresponding to the largest eigenvalue of $\left(\mathbf{A}_{n}^{\dagger} \mathbf{A}_{n}\right)^{-1} \mathbf{A}_{p}^{\dagger} \mathbf{A}_{p}$. Efficient algorithms for obtaining the optimal solution can be found in [19].

Remark: If the objective function in (11) is used instead, the optimization becomes

$$
\text { maximizing } \mathbf{t}^{\dagger} \mathbf{A}_{p}^{\dagger} \mathbf{A}_{p} \mathbf{t} \text {, subject to } \mathbf{t}^{\dagger}\left(\mathbf{A}_{m}^{\dagger} \mathbf{A}_{m}+\mathbf{A}_{n}^{\dagger} \mathbf{A}_{n}\right) \mathbf{t}=1 \text {. }
$$

In this case, the optimal $\mathbf{t}$ is the eigenvector corresponding to the largest eigenvalue of $\left(\mathbf{A}_{m}^{\dagger} \mathbf{A}_{m}+\mathbf{A}_{n}^{\dagger} \mathbf{A}_{n}\right)^{-1} \mathbf{A}_{p}^{\dagger} \mathbf{A}_{p}$.

\section{A. Efficient Formulation of $\phi$}

To formulate the objective function, we need to compute $\mathbf{A}_{p}$ and $\mathbf{A}_{n}$, i.e., compute $\mathbf{W} \overline{\mathbf{R}}$ in (13). Observe that $\overline{\mathbf{R}}$ is a Toeplitz matrix with entries drawn from the $(M+Q) \times 1$ vector $\overline{\mathbf{r}}$

$$
\overline{\mathbf{r}}=\left(\begin{array}{c}
\frac{1}{B} \sum_{i=1}^{B} r(i N+L-Q) \\
\frac{1}{B} \sum_{i=1}^{B} r(i N+L+1-Q) \\
\vdots \\
\frac{1}{B} \sum_{i=1}^{B} r(i N+N-1)
\end{array}\right) \text {. }
$$

$$
\mathbf{R}_{n}=\left(\begin{array}{cccc}
r(n N+L) & r(n N+L-1) & \cdots & r(n N+L-Q) \\
r(n N+L+1) & r(n N+L) & \cdots & r(n N+L+1-Q) \\
\vdots & \vdots & \ddots & \vdots \\
r(n N+N-1) & r(n N+N-2) & \cdots & r(n N+N-1-Q)
\end{array}\right)
$$


1. Collect $B$ blocks of received signal and compute the average received vector $\overline{\mathbf{r}}$ given in (14).

2. Compute $\mathbf{A}_{p}$ and $\mathbf{A}_{n}$ by first computing $\mathbf{A}=\mathbf{W} \overline{\mathbf{R}}$ using sliding window FFT.

3. Compute $\left(\mathbf{A}_{n}^{\dagger} \mathbf{A}_{n}\right)^{-1} \mathbf{A}_{p}^{\dagger} \mathbf{A}_{p}$.

4. Obtain the optimal $\mathbf{t}$ by computing the eigen vector corresponding to the largest eigen value of $\left(\mathbf{A}_{n}^{\dagger} \mathbf{A}_{n}\right)^{-1} \mathbf{A}_{p}^{\dagger} \mathbf{A}_{p}$.

Fig. 3. Design procedure for the proposed TEQ optimization.

The matrix $\overline{\mathbf{R}}$ can be obtained by computing the average received vector $\overline{\mathbf{r}}$. To compute $\mathbf{A} \triangleq \mathbf{W} \overline{\mathbf{R}}$ is to apply $M$-point DFT on each column of $\overline{\mathbf{R}}$. To efficiently compute $\mathbf{A}$, we observe the following property of $\overline{\mathbf{R}}$ : the last $M-1$ elements of the $(i+1)$ th column are the same as the first $M-1$ elements of the $i$ column. The computation of $\mathbf{A}$ can be done using sliding window fast Fourier transform as follows. In particular, the $(k, i)$ th element and the $(k, i+1)$ th element of $\mathbf{A}$ are related by [21]

$[\mathbf{A}]_{k, i}=W^{-k}\left([\mathbf{A}]_{k,(i+1)}-\overline{\mathbf{r}}_{Q-i-1}\right)+\overline{\mathbf{r}}_{Q+M-1-i} W^{k(M-1)}$.

Given $[\mathbf{A}]_{k,(i+1)}$, we can compute $[\mathbf{A}]_{k, i}$ using two complex multiplications and two complex additions. Therefore, to compute $\mathbf{A}$, we only need to apply $M$-point DFT on the last column of $\overline{\mathbf{R}}$ to obtain the last column of $\mathbf{A}$, and the other columns of A can be computed efficiently. We summarize the design procedure for TEQ optimization in Fig. 3.

\section{B. Tone Decimation}

In the objective function in (10), we have used all pilot tones and all null tones, with $\overline{\mathbf{u}}_{p}$ denoting the collection of averaged output on all pilot tones and $\overline{\mathbf{u}}_{n}$ denoting the collection of averaged output on all null tones. Suppose the dimension of $\overline{\mathbf{u}}_{p}$ is $M_{p}$ and the dimension of $\overline{\mathbf{u}}_{n}$ is $M_{n}$. The complexity of TEQ design in the above procedure depends on $M_{p}$ and $M_{n}$ as $\mathbf{A}_{p}$ is of size $M_{p} \times Q$ and $\mathbf{A}_{n}$ is of size $M_{n} \times Q$. To reduce computation, we can leave out some of the pilot tones and null tones in the formulation of objective function. For example, we can use only half the pilot tones and null tones; $\overline{\mathbf{u}}_{p}$ contains only one of every two pilot tones and $\overline{\mathbf{u}}_{n}$ contains only one of every two null tones. The dimensions of $\overline{\mathbf{u}}_{p}$ and $\overline{\mathbf{u}}_{n}$ will be halved. In this case the new $\mathbf{A}_{p}$ contains only the even rows of the original $\mathbf{A}_{p}$, and similarly, the number of rows in $\mathbf{A}_{n}$ will be reduced by half. More generally, we can choose to decimate the pilot tones and null tones by a number $D$. Experiments given in the next section show that the decimation of pilot and null tones leads to only a minor performance degradation.

\section{SimUlation EXAMPLES}

In the simulations, the DFT size is 8192 , cyclic prefix length is 640 , sampling rate is $35.328 \mathrm{MHz}$, and $B=20$ blocks of VDSL symbols are used. We consider downstream transmission, so the upstream tones are null tones. Symmetric VDSL transmission is assumed. The tones used for downstream transmission are 33-870 and 1206-1970 [2]. The pilot tones, as described in Section II, are the even tones and the tones that are multiples of ten plus nine. The noise is composed of additive

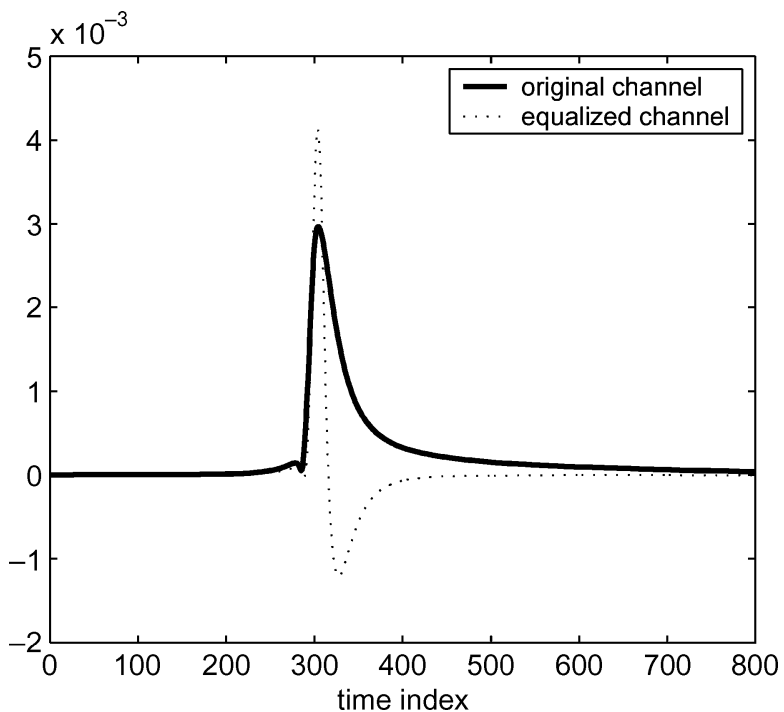

Fig. 4. The impulse responses of the original VDSL loop 7 and the equalized channel.

white Gaussian noise and crosstalk (FEXT and NEXT) generated from ten ADSL disturbers, 16 ISDN users, 4 HDSL, and 20 VDSL disturbers as described in the VDSL testing environment given in [2]. The transmission power is $14.5 \mathrm{dBm}$. The channels used in the simulations are test loops 1-7 from [2]. The analog front-end bandpass filter is not included. We assume symbol synchronization has been properly established.

Example 1-SIR Simulations: We will first use loop 7, the longest test loop in [2], as an example. Fig. 4 shows the impulse response of the channel. A commonly used measure for TEQ is SIR. Let the equalized channel be $h(n)$; then SIR is defined as

$$
\operatorname{SIR}=\max _{\Delta} 10 \log \frac{\sum_{n=\Delta}^{\Delta+L}|h(n)|^{2}}{\sum_{n \notin\{\Delta, \Delta+1, \ldots, \Delta+L\}}|h(n)|^{2}}
$$

where $L$ is the length of cyclic prefix. The SIR of the original channel is $35.7 \mathrm{~dB}$.

We compute the average received vector $\overline{\mathbf{r}}$ and matrices $\mathbf{A}_{p}$, $\mathbf{A}_{n}$ as described in Section IV, and the optimal TEQ of 20 taps for the objective function in (10) is computed. The impulse response of the resulting TEQ is given in Fig. 5. It has the property of being approximately antisymmetric as detailed in [20]. The magnitude response of the TEQ in Fig. 5 is as shown in Fig. 6. Convolving the optimized TEQ with the original channel, we get the equalized channel as shown in Fig. 4. The SIR of the equalized channel is $58.8 \mathrm{~dB}$. We can see from the magnitude response in Fig. 6 that the zeros of the TEQ are mostly placed in the upstream bands as the underlined application is downstream 


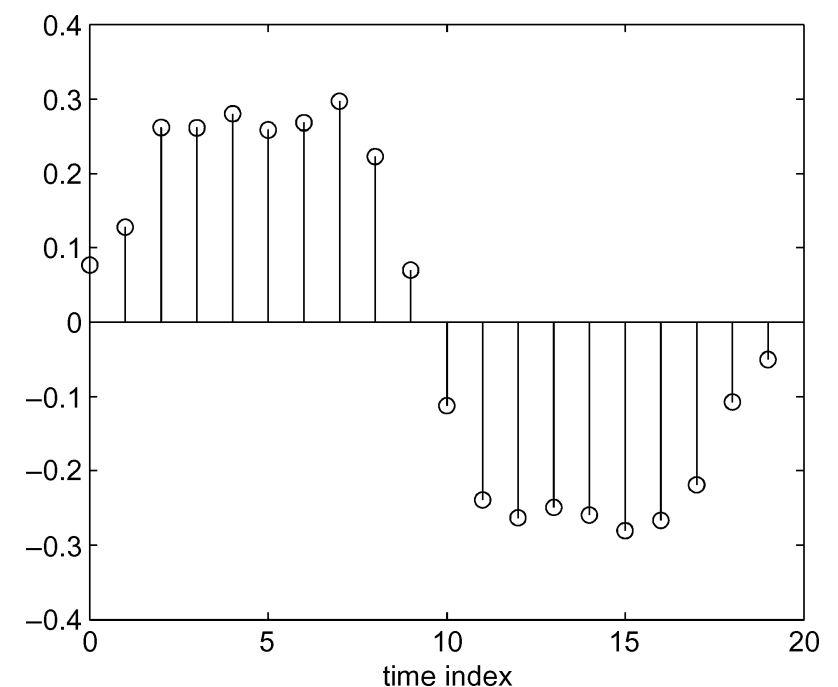

Fig. 5. The impulse response of the TEQ optimized using the proposed semiblind eigen approach.

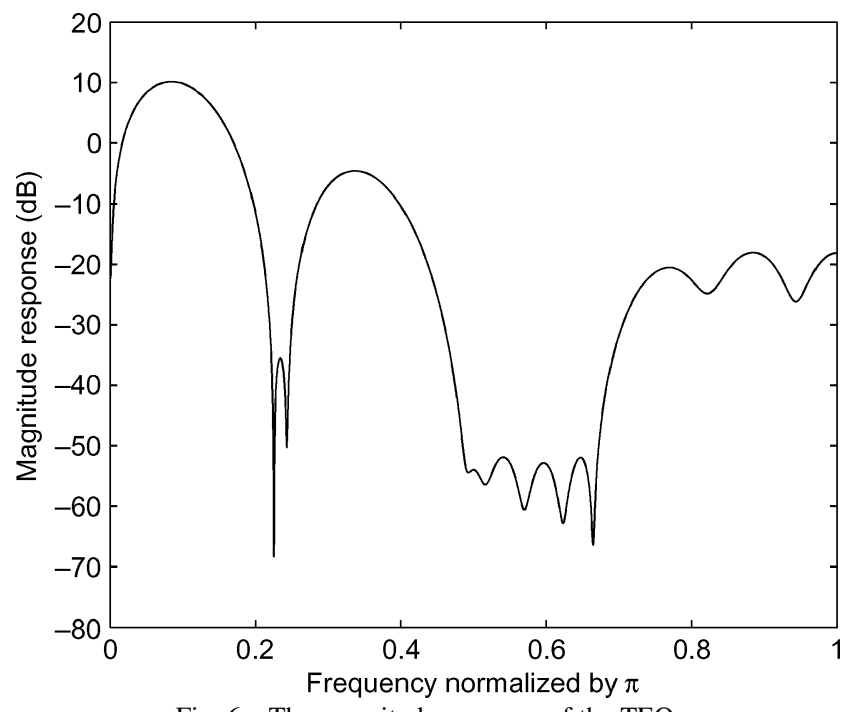

Fig. 6. The magnitude response of the TEQ.

transmission. Fig. 7 shows the magnitude responses of the original channel and the equalized channel.

Using the proposed TEQ design method [both objective functions (10) and (11)], we list in Table I the SIR of the equalized channels for the test loops listed in [2]. The test loops VDSL 1-4 used are long loops of length $4500 \mathrm{ft}$. The objective function in (11), which incorporates the interference on message tones, gives a better SIR performance. For comparison, we have also listed the SIRs of the equalized channels using maximum shortening signal-to-noise ratio (MSSNR) [3] and MERRY [14]. MSSNR is known to maximize time-domain SIR, while MERRY is known to provide a very good tradeoff between performance and complexity. For MSSNR, the channel impulse response is given but the channel noise statistics is not incorporated. In the training of TEQ for MERRY, we also use 20 blocks, which is equivalent to around 17000 iterations as MERRY performs one iteration for every sample. In all cases, the TEQ is of 20 taps. We can see that the proposed TEQ design can achieve good SIR for all test loops. In our proposed method, we consider the interference in the null tones rather

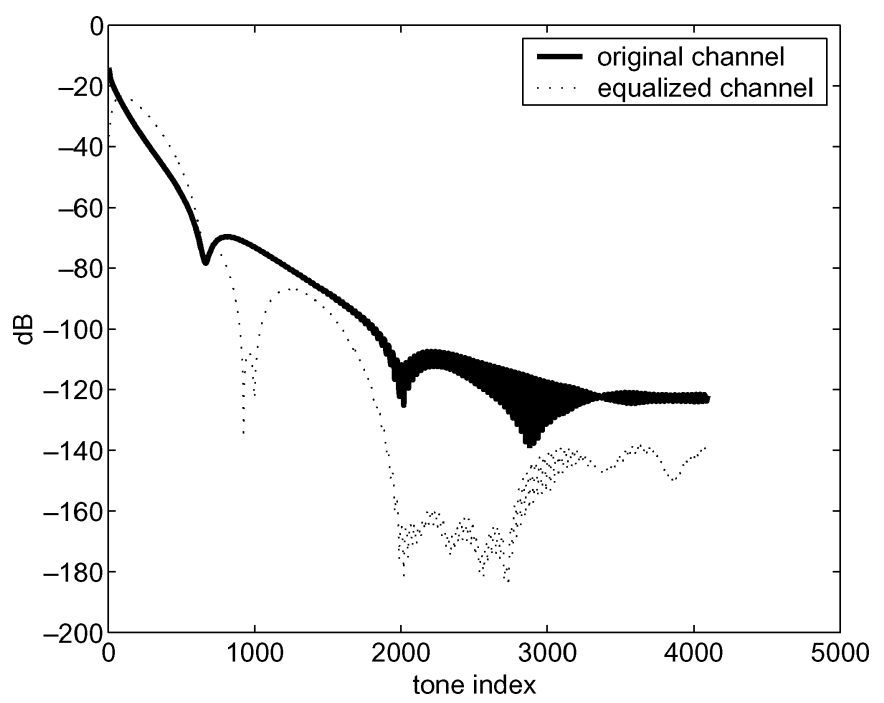

Fig. 7. The magnitude responses of the original channel and the equalized channel.

TABLE I

Comparison of SIR (dB) PeRformances on VDSL LoOPS

\begin{tabular}{|c|c|c|c|c|}
\hline \hline VDSL loop & proposed(10) & proposed(11) & MERRY & MSSNR \\
\hline VDSL-1L & 63.1 & 71.1 & 49.9 & 96.5 \\
\hline VDSL-2L & 57.4 & 68.2 & 52.0 & 84.8 \\
\hline VDSL-3L & 62.7 & 65.0 & 46.7 & 86.8 \\
\hline VDSL-4L & 50.4 & 41.8 & 32.4 & 73.6 \\
\hline VDSL-5 & 90.6 & 95.0 & 74.6 & 98.8 \\
\hline VDSL-6 & 67.5 & 76.8 & 53.8 & 92.1 \\
\hline VDSL-7 & 58.8 & 58.2 & 40.4 & 79.8 \\
\hline \hline
\end{tabular}

than all the tones and there is no explicit optimization of SIR in the time domain. The results demonstrate that such a design leads to a very good shortening effect and very good SIR in the time domain. Note that the SIR obtained from the proposed TEQ method is not as high as the MSSNR method. However, minimization of SIR in the time domain in the MSSNR method is to minimize SIR for all tones regardless of whether the tones are used for transmission or not; a smaller total SIR does not necessarily mean a higher transmission rate.

Example 2-Bit Rate Simulations: With the TEQ in place, we measure subchannel SINR, denoted by $\operatorname{SINR}_{k}$. The number of bits allocated for the $k$ th subchannel is $b_{k}=\left\lfloor\log _{2}(1+\right.$ $\left.\left(\operatorname{SINR}_{k} / \Gamma\right)\right)$ ], where $\Gamma$ is the SNR gap corresponding to an error rate of $10^{-7}$. The transmission rate is equal to

$$
\text { sampling rate } \cdot \frac{M}{N} \sum_{k} b_{k} \text {. }
$$

The SIR and bit rate performance with tone decimation for loop 7 is tabulated in Table II. The number $D$ is the decimation ratio. For example, with $D=1$, there is no tone decimation, and with $D=2$, the pilot tones and null tones used are decimated by two. We can see that good SIR performance can be obtained with $D$ up to eight. There is no noticeable effect on bit rates for $D$ up to 32 .

We calculate the transmission bit rates for the seven test loops using the TEQ designed in Example 1. We list in Table III the bit rates of the equalized channels for the seven test loops. We have also listed the bit rates when the TEQ is designed using 
TABLE II

THE SIR AND BIT RATE PERFoRMANCES WITH TONE DECIMATION FOR VDSL LOOP 7, WHERE THE NUMBER $D$ DENOTES THE DECIMATION RATIO

\begin{tabular}{|c|c|c|c|c|c|c|}
\hline \hline $\mathrm{D}$ & 1 & 2 & 4 & 8 & 16 & 32 \\
\hline SIR (dB) & 58.8 & 59.7 & 59.7 & 56.4 & 53.7 & 49.0 \\
\hline bit rate (Mbits/sec) & 29.0 & 29.0 & 29.0 & 29.0 & 29.0 & 29.0 \\
\hline \hline
\end{tabular}

TABLE III

COMPARISON OF BIT RATES (Mbits/s) ON VDSL LOOPS

\begin{tabular}{|c|c|c|c|c|}
\hline \hline VDSL loop & proposed(10) & proposed(11) & MERRY & MSSNR \\
\hline VDSL-1L & 46.1 & 49.9 & 47.1 & 41.2 \\
\hline VDSL-2L & 41.5 & 43.7 & 42.2 & 32.0 \\
\hline VDSL-3L & 40.5 & 42.7 & 41.2 & 35.3 \\
\hline VDSL-4L & 23.3 & 23.3 & 23.4 & 19.5 \\
\hline VDSL-5 & 94.5 & 95.1 & 94.7 & 81.6 \\
\hline VDSL-6 & 51.3 & 55.3 & 52.5 & 46.6 \\
\hline VDSL-7 & 29.0 & 29.9 & 29.2 & 24.5 \\
\hline \hline
\end{tabular}

the MSSNR and MERRY method as in Example 1. In all cases, the TEQ is of 20 taps. Our proposed method is slightly better than MERRY with the objective function in (11) and slightly worse with the objective function in (10). When compared with MSSNR, the proposed TEQ design can achieve a higher bit rate for all test loops. This is because MSSNR maximizes SIR in the time domain, disregarding the frequency response of the equivalent channel. In this case, zeros in the transmission bands are often incidentally introduced by TEQ. These zeros usually lead to a significant loss in tone SNR and hence final bit rate. Our proposed method formulates the objective function in the frequency domain to take advantage of the fact that the VDSL system is frequency-division duplexing. SIR is maximized in the frequency domain. As a result, the frequency response of the equalized channel is implicitly taken into account and a better bit rate is achieved.

\section{CONCLUSION}

In this paper, we propose a semiblind TEQ design for VDSL systems using an eigen filter approach. Unlike earlier eigen designs, the proposed method does not require an estimate of the channel impulse response nor channel noise statistics. This saves computations as well as training time needed. The objective function is formulated in the frequency domain to take advantage of the frequency-division duplexing property of the VDSL system. The training symbols of VDSL systems are exploited to maximize signal power in the transmission bands to interference in the null tones. Examples demonstrated that the proposed TEQ leads to very good channel shortening results and ISI can be significantly reduced. Due to the incorporation of signal power in transmission band, good subchannel SINR can be obtained. High bit rate can be achieved with only a small number of training symbols.

\section{REFERENCES}

[1] Asymmetric Digital Subscriber Lines (ADSL)-Metallic Interface, ANSI T1.413, 1998

[2] Very-High Speed Digital Subscriber Lines (VDSL)-Metallic Interface, ANSI T1.424, 2002.

[3] P. J. W. Melsa, R. C. Younce, and C. E. Rohrs, "Impulse response shortening for discrete multitone transceivers," IEEE Trans. Commun., vol. 44, pp. 1662-1672, Dec. 1996.
[4] N. Al-Dhahir and J. M. Cioffi, "Optimum finite-length equalization for multicarrier transceivers," IEEE Trans. Commun., vol. 44, pp. 56-64, Jan. 1996.

[5] G. Arslan, B. L. Evans, and S. Kiaei, "Equalization for discrete multitone transceivers to maximize bit rate," IEEE Trans. Signal Process., vol. 49, pp. 3123-3135, Dec. 2001.

[6] K. Van Acker, G. Leus, M. Moonen, and T. Pollet, "RLS-based initialization for per-tone equalizers in DMT receivers," IEEE Trans. Commun., vol. 51, Jun. 2003.

[7] K. Vanbleu, G. Ysebaert, G. Cuypers, M. Moonen, and K. Van Acker, "Bitrate maximizing time-domain equalizer design for DMT-based systems," IEEE Trans. Commun., vol. 52, pp. 871-876, Jun. 2004.

[8] C.-Y. Chen and S.-M. Phoong, "Bit rate optimized time-domain equalizers for DMT systems," in Proc. Int. Symp. Circuits Syst., 2003.

[9] I. Djokovic, "MMSE equalizers for DMT systems with and without cosstalk," in Conf. Rec. 31st Asilomar Conf. Signals, Syst. Comput., Nov. 1997.

[10] A. Tkacenko and P. P. Vaidyanathan, "A low-complexity eigenfilter design method for channel shortening equalizers for DMT systems," IEEE Trans. Commun., vol. 51, pp. 1069-1072, Jul. 2003.

[11] R. Lopez-Valcarce, "Minimum delay spread TEQ design in multicarrier systems," IEEE Signal Process. Lett., vol. 11, Aug. 2004.

[12] M. de Courville, P. Duhamel, P. Madec, and J. Palicot, "Blind equalization of OFDM systems based on the minimization of a quadratic criterion," in Proc. IEEE Int. Conf. Commun., Jun. 1996, vol. 3, pp. $1318-1322$

[13] B. Farhang-Boroujeny and M. Ding, "Design methods for time-domain equalizers in DMT transceivers," IEEE Trans. Commun., vol. 49, pp. 554-562, Mar. 2001

[14] R. K. Martin, J. Balakrishnan, W. A. Sethares, and C. R. Johnson, Jr., "A blind, adaptive TEQ for multicarrier systems," IEEE Signal Process. Lett., vol. 9, pp. 341-343, Nov. 2002.

[15] R. K. Martin, K. Vanbleu, M. Ding, G. Ysebaert, M. Milosevic, B. L. Evans, M. Moonen, and C. R. Johnson, Jr., "Unification and evaluation of equalization structures and design algorithms for discrete multitone modulation systems," IEEE Trans. Signal Process., to be published.

[16] Y.-P. Lin, L.-H. Liang, and S.-M. , "An semi-blind eigen approach to time-domain equalizer design for VDSL systems," in Proc. IEEE Int. Conf. Acoust., Speech, Signal Process., Mar. 2005, vol. 3, pp. 341-344.

[17] Y.-P. Lin and S.-M. Phoong, "Optimal ISI free DMT transceivers for distorted channels with colored noise," IEEE Trans. Signal Process., vol. 49, Nov. 2001

[18] G. H. Golub and C. F. Van Loan, Matrix Computations. Baltimore, MD: Johns Hopkins Univ. Press, 1996.

[19] R. K. Martin, K. Vanbleu, M. Ding, G. Ysebaert, M. Milosevic, B. L. Evans, M. Moonen, and C. R. Johnson, Jr., "Implementation complexity and communication performance tradeoffs in discrete multitone modulation equalizers," IEEE Trans. Signal Process., to be published.

[20] R. K. Martin, M. Ding, B. L. Evans, and C. R. Johnson, Jr., "Infinite length results and design implications for time-domain equalizers," IEEE Trans. Signal Process., vol. 52, pp. 297-301, Jan. 2004.

[21] K. Van Acker, G. Leus, M. Moonen, O. van de Wiel, and T. Pollet, "Per tone equalization for DMT-based systems," IEEE Trans. Commun., vol. 49, Jan. 2001

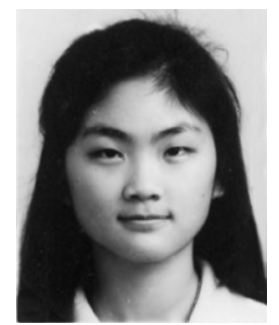

Yuan-Pei Lin (S'93-M'97-SM'03) was born in Taipei, Taiwan, R.O.C., in 1970. She received the B.S. degree in control engineering from the National Chiao-Tung University, Taiwan, in 1992 and the M.S. and Ph.D. degrees, both in electrical engineering, from the California Institute of Technology, Pasadena, in 1993 and 1997, respectively.

She joined the Department of Electrical and Control Engineering, National Chiao-Tung University, in 1997. Her research interests include digital signal processing, multirate filter banks, and signal processing for digital communication, particularly the area of multicarrier transmission.

Dr. Lin received the 2004 Ta-You Wu Memorial Award. She served as an Associate Editor for the IEEE TRANSACTIONS ON SIGNAL PROCESSING from 2002 to 2006. She is currently an Associate Editor for the IEEE TRANSACTIONS ON CIRCUITS AND SySTEMS-PART II: ANALOG AND DigitAL SigNAL PROCESSING, the EURASIP Journal on Applied Signal Processing and Multidimensional Systems, and Signal Processing. She is also a Distinguished Lecturer of the IEEE Circuits and Systems Society for 2006-2007. 


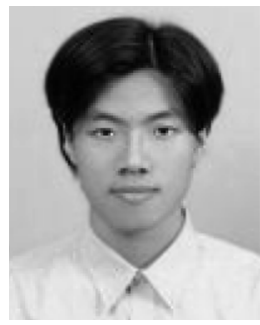

Li-Han Liang was born in Kaohsiung, Taiwan, R.O.C., in 1980. He received the B.S. degree in mechanical engineering and the M.S. degree in electrical and control engineering from National Chiao-Tung University, Hsinchu, Taiwan, in 2002 and 2004, respectively.

Since 2004, he has been with REALTEK Semiconductor Corp., where he participates in the system design for DVR servo systems. His research interest is signal processing for communications, particularly the area of multicarrier systems.

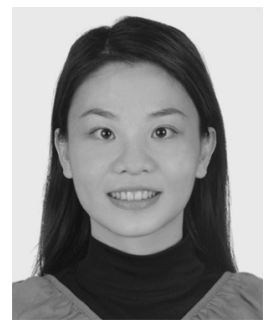

Pei-Ju Chung was born in Pingtung, Taiwan, R.O.C., in 1980. She received the B.S. degree in electrical engineering from I-Shou University, Kaohsiung, Taiwan, in 2003 and the M.S. degree in electrical and control engineering from National Chiao-Tung University, Hsinchu, Taiwan, in 2005.

Her research interests include digital signal processing and signal processing for digital communication.

Ms. Chung was an outstanding college youth of the China Youth Corps and an outstanding university youth of I-Shou University in 2002

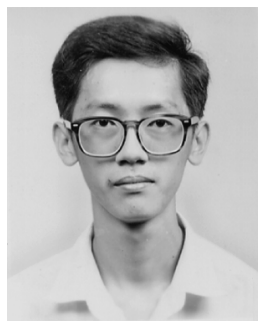

See-May Phoong (M'96) was born in Johor, Malaysia, in 1968. He received the B.S. degree in electrical engineering from National Taiwan University (NTU), Taipei, Taiwan, R.O.C., in 1991 and the M.S. and Ph.D. degrees in electrical engineering from the California Institute of Technology (Caltech), Pasadena, in 1992 and 1996, respectively.

He was with the Faculty of the Department of Electronic and Electrical Engineering, Nanyang Technological University, Singapore, from September 1996 to September 1997. In September 1997, he joined the Graduate Institute of Communication Engineering and the Department of Electrical Engineering, NTU, as an Assistant Professor, where since August 2001, he has been an Associate Professor. His interests include multirate signal processing, filter banks, and their application to communications.

Dr. Phoong is currently an Associate Editor for the IEEE TRANSACTIONS ON CiRCUITS AND SYSTEMS-PART I: FUNDAMENTAL THEORY AND APPLICATIONS. $\mathrm{He}$ previously was an Associate Editor for the IEEE TRANSACTIONS ON CIRCUITS AND SYSTEMS-PART II: ANALOG AND DigITAL SIGNAL PROCESSING (January 2002-December 2003) and IEEE Signal Processing LeTters (March 2002-February 2005). He received the Charles H. Wilts Prize (1997) for outstanding independent research in electrical engineering at Caltech. He also received the Chinese Institute of Electrical Engineering's Outstanding Youth Electrical Engineer Award (2005). 